\title{
LA SELECCIÓN NATURAL: LENGUAJE, MÉTODO Y FILOSOFÍA
}

\section{NATURAL SELECTION: LANGUAGE, METHOD, AND PHILOSOPHY}

\author{
Juan Ramón Álvarez* \\ Departamento de Psicología, Sociologia y Filosofia \\ Facultad de Filosofía y Letras \\ Universidad de León
}

RESUMEN: La selección natural (SN) es el principio explicativo de la teoría de la evolución de Darwin. Tras una puesta al día de la situación actual, este artículo estudia, en primer lugar, el doble lenguaje en que formuló su idea de la SN. En segundo lugar, se presenta la estructura del «extenso argumento» de El origen de las especies como la del circuito de la explicación científica, que unifica los procesos de descubrimiento y justificación, en términos de abducción y reconstrucción, respectivamente. En último lugar, se considera cómo SN juega el papel de principio explicativo de una teoría científica y de idea regulativa de una filosofía naturalista, que marca las líneas de un programa, cuyo desarrollo científico se ha producido en el conjunto de las ciencias biológicas. Pero sus consecuencias filosóficas y tecnológicas conducen también a planteamientos metafísicos y bioéticos de importancia.

Palabras Clave: Abducción, analogía, Darwin, explicación científica, filosofía, metáfora, selección natural.

ABSTRACT: Natural selection (NS) is the explanatory principle of Darwin's evolutionary theory. After updating the subject this paper analyses, in the first place, the twofold language in which Darwin expressed his idea of NS. Second,

* Correo-e: juan-ramon.alvarez@unileon.es 
the structure of the Origin of Species" «long argument» is displayed as the cycle of scientific explanation encompassing discovery and justification, respectively in terms of abduction and reconstruction. At the end it is shown how SN plays the role of an explanatory principle in a scientific theory as well as that of a regulative idea in a naturalistic philosophy, setting the main lines of a program whose development has been accomplished in biological sciences altogether. Yet its philosophical and technological implications also lead to remarkable metaphysical and bioethical approaches.

KeYwORDs: Abduction, analogy, Darwin, metaphor, natural selection, philosophy, scientific explanation.

Varios autores han entendido mal o han puesto objeciones al término Selección Natural. Algunos hasta han imaginado que la selección natural produce la variabilidad, aunque sólo implica la conservación de las variaciones que surgen y son beneficiosas para el ser en sus condiciones de vida [...] En el sentido literal de la palabra, indudablemente, selección natural es un término falso; pero ¿quién ha puesto alguna vez objeciones a los químicos que hablan de las afinidades electivas de los diferentes elementos? [...] Todos sabemos lo que significan e implican tales expresiones metafóricas, que, además, son casi siempre necesarias en pos de la brevedad. Del mismo modo, también es difícil evitar la personificación de la palabra Naturaleza; pero por Naturaleza entiendo solamente la acción conjunta y el producto de muchas leyes naturales, y por leyes la sucesión de hechos tal como son comprobados por nosotros. Familiarizándose un poco con los términos, tales objeciones superficiales quedarán olvidadas.

(Charles Darwin, El origen de las especies, 3..$^{a}$ edición y sucesivas)

\section{Introducción}

Deseo vano. Darwin escribió estas palabras en $1861^{1}$ — dos años después de la primera edición de $\mathrm{OS}^{2}$ - y las mantuvo en las ediciones posteriores. $\mathrm{Al}$ pare-

${ }^{1}$ Cf. Ruse $(2008,87)$.

${ }^{2}$ En lo que sigue utilizaré OS para abreviar $(O n)$ the Origins of Species (sexta edición, salvo indicación diferente) y OE para la traducción española de dicha edición (Darwin, 1985), que figura en la bibliografía, cuyo texto seguiré, a veces con ligeras modificaciones, como en este caso. Mi elección se basa en que Darwin la redactó con la vista puesta en las reacciones provocadas por las anteriores y en sus propias reflexiones posteriores. 
cer, la familiaridad con los términos no ha tenido lugar o no ha eliminado las objeciones a la idea de selección natural (SN): tanto las menos numerosas, pero más vehementes, que se plantean desde fuera y en contra de la teoría darwinia$\mathrm{na}^{3}$, como las más variadas e interesantes en los debates cruzados que tienen lugar dentro del propio programa darwinista ${ }^{4}$, entre las diferentes corrientes que se atribuyen la concepción más adecuada de la teoría de la evolución.

Volver otra vez sobre «la peligrosa idea de Darwin« (Dennett, 1995, dixit) tiene, a su vez, el peligro añadido de repetir o dar vueltas hasta el cansancio en torno a un tema que parece interminable, dada la creciente «industria cultural darwiniana» (Cf. Eldredge, 2009; Castrodeza, 2009). Para evitarlo, me guiaré por el texto de queja, reconocimientos, aclaración y esperanza del Darwin de 1861, que sirve aquí de introito 5 .

A juzgar por los numerosos e intensos debates sobre la SN desde 1859, es dudoso que las objeciones hayan sido meramente «superficiales». No lo han sido en la negación desde fuera — donde antiguos y nuevos creacionismos disimula-

3 Todos los antidarwinismos, en general y, en particular, los creacionismos y su versión edulcorada, la tesis del diseño inteligente — sin diseñador designado, porque quieren ponerse en paralelo con la selección sin seleccionador- que en los últimos años ha tenido mucha difusión. Sobre este último, Cf. Sarkar (2007) y Pievani (2009). Los creacionistas duros descalifican la teoría darwinista calificándola despectivamente de teoría, es decir de mera especulación. (Cf. Johnson, 1995). La iglesia católica hila algo más fino y, en las propias palabras del fenecido Papa Juan Pablo II, afirmaba que «Hoy [...] [1996] los nuevos conocimientos conducen a reconocer que la teoría de la evolución es algo más que una hipótesis» (Russell, R.J., Stoegen, W. R. \& Ayala, F.J. (eds.), 1998, 5). Los creacionistas protestantes duros consideran esto una concesión excesiva, pero es dudoso que una expresión tan rebajada de tono represente ni mucho menos una aceptación, aunque sea subordinándola a la creación originaria del dios cristiano que luego deja que el mundo ruede según causas segundas, expresables incluso en leyes de la naturaleza (creada). Pero este no el tema de este artículo.

${ }^{4}$ Uso «darwiniana» para referirme a la doctrina expresada en los escritos del propio Darwin y «darwinista» para todas las versiones que se consideran seguidoras o desarrollos actualizados del programa inicial trazado por Darwin. Para una consideración de estas variaciones terminológicas apoyada en la historia, Cf. Gayon (2009, 322 y ss.) y Quammen $(2007,12)$.

5 Texto del que parte y en que se apoya Young (1985, cap. IV; 1993), en el segundo de los cuales se vierten las siguientes afirmaciones. "Por tanto, la historia de la ciencia puede ser considerada no como una historia de los descubrimientos, sino como una historia de las metáforas. Esto forma parte de una forma de pensar en que vemos que la verdad se construye y no se encuentra» (Young, 1993, 8). 
dos no son «objeciones» ideológica y socialmente superficiales, porque está en juego el poder y el adoctrinamiento en la educación general, con sentencias de tribunales incluidas-, ni en las alternativas «internas» acerca del papel único, principal o complementario de la SN como principio explicativo de la evolución biológica.

\section{Malentendidos y objeciones: ciento cincuenta años de lucha por la existencia}

En el sesquicentenario de OS, volver a contar la historia de las vicisitudes de la teoría darwiniana, convertida en diferentes "darwinismos», puede ser reiterativo y, sobre todo, prematuro, porque se trata de algo vivo y coleante que, como las buenas series, continuará. Ello no obsta para que tenga la obligación, al menos, de dar testimonio de cómo andan las cosas a la fecha. Dejo para autores más pacientes las negaciones radicales desde las convicciones «profundas» de los creacionistas, desnudos o revestidos. La situación a la que se ha llegado dentro de los diferentes darwinismos que, casi todos, aseguran ser los verdaderamente darwinianos, es la culminación de una trayectoria con altibajos desde 1859, cuya historia detallada ha sido expuesta adecuadamente — eso sí, siempre con los sesgos correspondientes - por muchos autores. Descartada la lucha con los adversarios externos y la presentación de los eclipses y resplandores de esos ciento cincuenta años ${ }^{6}$, voy a tomar la cuestión como en los últimos años le ha ido a la idea de $\mathrm{SN}$ en el ámbito del darwinismo, total o parcialmente asumido. ¿Es el darwinismo una teoría en crisis? Una respuesta contundente y negativa la ha dado en España, el año pasado, Juan Moreno (Moreno, 2008), quien, con extensos conocimientos y datos abundantes, argumenta a conciencia que «los retos del darwinismo» han sido, van siendo y, con alta probabilidad, serán respondidos por las investigaciones biológicas (ecológicas, paleontológicas, genéticas, etc.) que cobran sentido con y dan contenido a esa idea darwiniana fundamental, la idea de SN en todas sus variantes: la diversidad es interna al dominio de la SN y fuera de ella — como en su día dijo Dobzhansky de la evolución— nada en biología tiene sentido. Moreno identifica darwinismo con SN: «[...] cuando mencione el término darwinismo, me estaré refiriendo a la teoría de la selección

\footnotetext{
${ }^{6}$ Un relato breve, claro y al día se encuentra en Gayon (2009). Véase también Bowler (2005).
} 
natural, la más original y controvertida de Darwin» (Moreno, 2008, 18) ${ }^{7}$. Por tanto, aquí no cabe aplicar la distinción darwiniano/darwinista, porque las objeciones intra muros, es decir, las de quienes sí distinguen entre darwiniano y darwinista (en diversos sentidos de este vocablo), son debates en los que

creemos que no se ha encontrado alternativa alguna sino meros retoques superficiales a lo que Charles Darwin escribió en una de las principales obras científicas jamás escrita, El origen de las especies. (Moreno, 2008,19).

Coincidencia casi literal con la esperanza de Darwin, las objeciones «superficiales» quedan fuera de juego si estamos bien familiarizados con los términos. Bien, pues el exhaustivo libro de Moreno consume, en esa familiarización actualizada, trescientas ochenta y una páginas. Una familiarización, por otra parte, que se presenta como una refutación de aquellas objeciones que no pretenden ser meros retoques, sino modificaciones importantes, no tanto de la teoría darwiniana, que no es el contrincante directo, sino de versiones darwinistas posteriores —entre y sobre todo la "síntesis moderna» de los treinta a cuarenta (Cf. Huxley, 1965)—, con sus prolongaciones de los setenta a noventa con Dawkins y Dennett como valedores, y más cerca Conway-Morris, Carroll y, por extensión, el propio Moreno. Como figuras sobresalientes que, aun aceptando la teoría de la $\mathrm{SN}$, ponen reparos a su acción, su eficacia y su extensión (Cf. Gould, 2002, 14-15), desfilan, llevando cada cual lo suyo, Gould, Eldredge, Lewontin, Goodwin, Kauffman, Jablonka, etc., (Cf. Moreno, 2008, 343), en la medida en que todos y cada cual a su manera consideran — reconociendo la importancia del Darwin de la esperanza puesta en la familiarización semántica- que la SN no es el único principio explicativo de la historia de la vida, aunque sea el principal y, en el peor de los casos, requiera principios no sólo complementarios, sino incluso previos. Sin duda, el antagonista principal es, como paleontólogo, Gould, en concreto el de la obra final y testamentaria (Gould 2002), considerado como quien, pretendidamente desde dentro, altera la continuidad del darwinismo, amparándose en una vuelta al proyecto darwiniano, que culmina en una teoría «revisionista».

${ }^{7}$ Lo que es coherente con que en el Glosario contenido en el libro no figure la expresión «selección natural», sino "darwinismo»: «teoría de Darwin sobre la evolución como resultado de la selección natural de variación fenotípica heredable» (Moreno, 2008, 348). 
La oposición está clara. Para unos, la SN es un principio omnicomprensivo o, por el contrario, para otros es un principio importante que no puede caminar solo en la ruta explicativa de la evolución biológica ${ }^{8}$. Moreno $(2008,344)$, en representación de los primeros, aboga por y bosqueja «[l] a nueva Síntesis darwinista del siglo XXI: Biología del Desarrollo, Ecología Evolutiva y Genética, provistas de herramientas moleculares, prometen los mayores avances en el estudio de la evolución por la selección natural, la única descubierta hasta la fecha.» Otra «nueva» síntesis, que se añade a las «síntesis inacabadas». Pero hay, además de los heterodoxos anteriores, «nuevas síntesis en marcha». Señalaré sólo una, la que complementa, en teoría de la evolución, la SN con la construcción de nichos $(\mathrm{CN})$,

el proceso por el cual los organismos modifican su nicho propio y/o el de otros, a través de su metabolismo, sus actividades y sus elecciones [...] Los defensores de la construcción de nichos [...] intentan explicar la complementariedad adaptativa del organismo y el medio en términos de interacciones dinámicas recíprocas entre los procesos de selección natural y construcción de nichos» (Laland et al, 2009, 196).

Pero en un libro tan exhaustivo como el de Moreno, no podía faltar y estar recogida, en beneficio de la SN, la CN. Y lo está desde el primer capítulo, porque precisamente Moreno reenfoca la «síntesis del siglo XXI» desde la ecología como «historia natural consciente de sí misma». La CN en un tiempo $t$ es resultado de la acción de los organismos seleccionados en un tiempo $t$ - 1 , es decir, «es el producto de la selección natural previa» (Moreno, 2008, 29). Pero esto constituye una diferencia fundamental. Mientras la teoría de la $\mathrm{CN}$ establece un circuito de realimentación entre los procesos de $\mathrm{SN}$ y de $\mathrm{CN}$ - circuito que se considera siempre in medias res_-, la tesis de Moreno es que la SN está determinando desde atrás la $\mathrm{CN}$ y, regresivamente, en el límite, se llegaría a una selección originaria, como consecuencia reflexiva con la que no se cuenta, pero que es lo que

${ }^{8}$ No entro ahora en el llamado darwinismo universal (Cf. Dawkins, 1983, Dennett, 1995) ni en la ya antigua extrapolación al problema del origen de la vida del principio de la SN, produciendo una teoría de la evolución generalizada, frente a la darwiniana — restringida - que no considera el origen de la vida desde el mecanismo de la selección natural (Cf. Álvarez, 1988a, 77 y ss.; Orgel, 1979).

9 Es una manera de hacer de la ecología la base de la teoría de la evolución, frente a los que considera sus principales antagonistas: los paleontólogos, que a juicio del autor presentan una versión deformada de la teoría de la evolución por selección natural. 
hace diferente la ecología evolucionista de Moreno (2008). Pero esa selección originaria establece barreras conceptuales derivadas directamente de los fundamentos metafísicos del neodarwinismo (Cf. Laland et al, 2009, 195) que, según se afirma, los tiene.

Parece, pues, que la esperanza darwiniana es imposible de cumplir mientras exista un vaivén entre estas dos tendencias, a menos que los problemas, los nuevos resultados y las nuevas interpretaciones dejen de producirse. Pero esa expectativa no parece en absoluto realista. Volvamos a Darwin.

\section{Metáforas y falsedad: el doble lenguaje y las analogías darwinianas.}

Dentro de la erudición darwiniana existe una producción renovada acerca de las metáforas y analogías de Darwin. Aquí hay de todo. Está la metáfora mayor, la expresión «selección natural», a la que su propio autor dedicó dos calificaciones extremas: «mi teoría», como la llamó desde muy pronto, hasta «término falso», tal como figura en el texto del comienzo. También son fundamentales las metáforas asociadas: «lucha por la existencia» (OS, cap. III) y «supervivencia de los más aptos» (sustitutiva de "selección natural», OS, cap. IV, a partir de la quinta edición). Las diversas analogías empleadas se unen a las metáforas en la articulación de un doble lenguaje en el cual están formuladas las ideas fundamentales de la teoría darwiniana: en el primer capítulo de OS se introduce la llamada «selección artificial» $(\mathrm{SA})^{10}$, que sirve de modelo (según otros, caso, experimento, etc., vid. infra) de la SN, de la que se ocupan los capítulos II, III y IV de OS. Como es sabido, Darwin publicó, urgido por el codescubrimiento de Wallace, un «resumen» de una obra proyectada que habría de llamarse La selección natural. Esta obra nunca se completó y en la «Introducción» a su presunta primera parte (Darwin 1868, Vol. I, 1-14), que coincide temáticamente con el capítulo I de OS, al proyecto de la obra añade dos partes más: una segunda obra en que trataría de la «variación de los organismos en estado de naturaleza, de la lucha

10 Uso «selección artificial» para referirme exclusivamente a los procedimientos de criadores de animales y plantas que Darwin consideró tanto en OS como en (Darwin, 1868). La expresión ha llegado a utilizarse para procedimientos biotecnológicos que intervienen en los genomas y también para las simulaciones informáticas dentro del campo de la llamada vida artificial. 
por la existencia y del principio de selección natural» (Ibid. , 8) $)^{11}$, y una tercera donde se tratarían las dificultades que se oponen a la teoría, indicando de forma poco precisa e inclusiva prácticamente el resto de OS (Ibid.).

Con independencia de que Darwin haya escrito OS hacia adelante o hacia atrás (Cf. Sober, 2009) $)^{12}$, de hecho Darwin eligió anteponer la teoría de la SN a la del ancestro común, poniendo por delante el orden causal al orden de la evidencia: «la obra sigue el orden causal correcto, pero está al revés en el orden evidencial» (Ibid, 65) ${ }^{13}$. A continuación trataré la teoría causal de la selección natural tal como figura en los primeros cuatro capítulos de OS, que incluyen la selección artificial como modelo de la natural, y las metáforas asociadas, distinguiendo la teoría casual de la SN del llamado principio de la $S N$, que Darwin formuló así en capítulo IV de OS:

A esta conservación de las variaciones y diferencias individualmente favorables y la destrucción de las que son perjudiciales, la he llamado selección natural o supervivencia de los más aptos (OS, 63; OE, 116).

A esta formulación se le ha llamado interpretación de la SN como consecuencia o efecto, por oposición a su interpretación como fuerza (Cf. Brunnander, 2005, 231). A pesar de la importancia que ha tenido la interpretación de SN como fuerza (Cf. especialmente Sober, 1993), prefiero oponer a la interpretación como consecuencia o efecto la interpretación causal, que atraviesa los primeros cuatro capítulos de OS.

En Álvarez (2000) traté el tema de las metáforas asociadas a la SN, así como algunas analogías importantes. Me basaré en aquel enfoque puesto al día con estudios posteriores sobre el tema, y modificado significativamente en sus conclusiones.

La consideración de SA como modelo de SN se basa en entender la relación de modelo como una relación analógica de proporcionalidad. Una relación entre

\footnotetext{
11 No hay referencia en este texto al capítulo V.: «Leyes de la variación».

12 Este artículo adquiere su sentido de la exposición elaborada en Sober (2008).

13 En esto Darwin no hizo sino mantener el orden seguido en el Bosquejo de 1842 y del Ensayo de 1848 (Cf. Eldredge, 2009, 156-180).
} 
cuatro términos, tales que $\mathrm{A}$ es modelo de $\mathrm{B}$ ssi existen relaciones en A que corresponden a las relaciones en $\mathrm{B}^{14}$.

Darwin utilizó SA como modelo de SN, en la medida en que los procesos (realmente procedimientos, acciones humanas) de SA dependen de y se apoyan en mecanismos subyacentes, desconocidos por él, que tienen lugar también en la naturaleza, con independencia de la acción humana. Los procedimientos de SA controlan desde fuera, canalizan, mecanismos en cuyo funcionamiento interno no intervienen ${ }^{15}$. La analogía darwiniana tiene la forma:

$$
\text { SA : SN :: Mec(SA): Mec(SN). }
$$

Pero la igualdad $\mathrm{Mec}(\mathrm{SN})=\mathrm{Mec}(\mathrm{SA})$ y la menor extensión de $\mathrm{SA}$, hacen que SA pase a ser un subconjunto propio de SN (SA İ SN), por lo cual se establece un homomorfismo (una inyección que conserva la estructura) de SA en SN. A este respecto son sumamente significativas las palabras del propio Darwin en el capítulo XV de OS («Recapitulación y conclusiones»):

[...] En estado doméstico vemos mucha variabilidad producida, o por lo menos estimulada, por el cambio de condiciones de vida; pero con frecuencia de una manera tan oscura que estamos tentados a considerar las variaciones como espontáneas. La variabilidad está regida por muchas leyes complejas [...] La variabilidad no es producida realmente por el hombre; el hombre tan sólo expone, y sin intención, los seres orgánicos a nuevas condiciones de vida, y luego la naturaleza obra sobre la organización y la hace variar. Pero el hombre puede seleccionar, y selecciona, las variaciones que le presenta la naturaleza, y las acumula así de la manera deseada. Así el hombre adapta a los animales y plantas a su propio beneficio o gusto. Puede hacer esto metódicamente,

${ }^{14}$ Idea claramente formulada en un opúsculo de Leibniz, en el que se define la noción de expresión. "Se dice que expresa una cosa aquello en lo que hay relaciones (habitudines) que responden a las relaciones de la cosa que ha de expresarse [...] no es necesario que lo que expresa sea semejante a la cosa expresada, con tal de que se conserve alguna analogía entre las relaciones.» (Leibniz, 1678, 263-264).

15 Utilizo aquí la distinción empleada por Sanmartín (1987, 27 y ss.) entre los procedimientos que permiten canalizar procesos, cuyos mecanismos subyacentes se desconocen, conforme a los fines de sus controladores (los técnicos, los artesanos), por oposición a los procedimientos de intervención que alteran los mecanismos subyacentes. 
o puede hacerlo inconscientemente, conservando a los individuos que le son más útiles o agradables, sin intención de modificar las castas. [...] Este proceso inconsciente de selección ha sido el agente principal en la formación de las razas domésticas más distintas y útiles.

No hay motivo alguno para que los principios que han obrado tan eficazmente en estado doméstico, no hayan obrado también en estado de naturaleza. (OS 410-411; OE, 460-61. Las cursivas son mías).

Sterrett (2002), ha contribuido significativamente a establecer la estructura fina de esta analogía mostrando en detalle que existe una correspondencia entre la SA metódica y el principio de divergencia en la naturaleza, así como entre la SA inconsciente y el principio de extinción en la naturaleza (Cf. Sterrett, 152) ${ }^{16}$.

Ahora la analogía está vista desde SN, que absorbe a SA que, al principio, sirvió de modelo de la primera y que, una vez cumplida su misión, se convierte en un caso de aquélla. Esto permite recuperar el planteamiento de Ginnobili (2009), quien asimila SA (y también la selección sexual) a un caso de SN, que sirve para su contrastación: «me parece que la selección artificial tiene un papel importantísimo en el contexto de justificación» (Ibid., 6) ${ }^{17}$. Como se verá más adelante, lo que empieza por ser un modelo en el contexto de descubrimiento, termina siendo un caso en el contexto de justificación: ser modelo o ser caso es,

${ }^{16}$ Contemplada desde el capítulo IV («Selección natural») la estructura de la analogía se resume como sigue: «1. Así como el principio de la divergencia en la naturaleza produce [...] (i.e, hace surgir) la diversificación de las variedades que terminan por convertirse en nuevas especies en la selección natural, así el principio de divergencia en las prácticas humanas de cría [...] produce la diversificación de las variedades que llegan a ser cada vez más distintas unas de otras en la selección artificial. 2. Así como el principio de extinción en la naturaleza [...] da como resultado especies adaptadas con mayor perfección a sus lugares en la naturaleza, así el principio de extinción en las prácticas de cría de los criadores humanos [...] dan como resultado crías cada vez más "perfectas». 3. Así como estos dos principios obran conjuntamente en la selección natural para producir la estructura ramificada de un "Árbol de la Vida", que da como resultado especies más numerosas y mejor adaptadas, así estos dos principios obran conjuntamente en la selección artificial para producir variedades más ampliamente diferenciadas y más «mejoradas». (Sterrett, 2002, 164. Las cursivas son de la autora)

17 «[...] la relación entre selección natural y selección artificial no es sólo de analogía [...] Yo sostengo algo más fuerte. La selección artificial, al menos la consciente, es una versión experimental de la selección natural. Lo que llevaba a cabo Darwin con las producciones domésticas eran contrastaciones de su teoría» (Ginnobili, 2009, 11). 
por tanto, una cuestión contextual. Sobre esta analogía la literatura es abundante, pero a los efectos de mi argumentación lo que antecede es suficiente ${ }^{18}$.

En el capítulo I se establece que la acción humana de selección (metódica o inconsciente), aplicada a las variaciones existentes, da como resultado la aparición de nuevas variedades que divergen de las existentes o que terminan sustituyéndolas por mejora (Cf. Sterrett, 2002). El esquema está claro. Dadas las variaciones, por medio de la acción de SA sobre ellas, se producen por acumulación efectos positivos — nuevas variedades se añaden a las existentes - y negativos — nuevas variedades sustituyen las anteriores, que se extinguen. La analogía entre SA y SN tiene su desarrollo en los tres capítulos siguientes. El capítulo dos sirve de puente para pasar de la variación en estado doméstico a la variación en estado de naturaleza. Asegurada una gran cantidad de variación en la naturaleza, el desarrollo de la analogía requiere una «acción de la naturaleza» que produzca resultados análogos a los de SA. En la expresión "acción de la naturaleza», la naturaleza parece tomar la apariencia de un agente, lo que preocupa al Darwin del texto inicial: «es difícil evitar la personificación de la palabra Naturaleza» (OS, 63; OE, 116). En el capítulo III corresponde tratar esa «acción de la naturaleza» y en el capítulo IV sus resultados.

Si Darwin afirma en el texto del comienzo que la expresión «selección natural», en sentido literal, es falsa, porque es metafórica, también lo son las metáforas asociadas «lucha por la existencia» $\mathrm{y}$ «supervivencia de los más aptos». He distinguido el principio de SN de la teoría causal de SN (que hago coincidir con los primeros cuatro capítulos de OS). "La expresión «lucha por la existencia» titula el capítulo III de ese bloque causal y también "en sentido literal» sería falsa. «Lucha por la existencia», expresión tomada de Malthus, es la denominación que toma esa acción de la naturaleza sobre las variaciones dadas. El siguiente texto de Darwin atestigua el doble lenguaje metafórico (falso) y literal (verdadero), respecto de esa "acción de la naturaleza», a la que llama «lucha por existencia»:

Debo hacer constar que empleo este término en un sentido amplio y metafórico, que incluye la dependencia de un ser respecto de otro, y - lo que es más importante- incluye no sólo la vida del individuo, sino también el éxito al

18 Valgan estas menciones recientes. Wilner (2006) y Ginnobili (2009) la consideran un experimento, alentados por una declaración de Darwin $(1868,3)$ en ese sentido. Gildenhuys (2004) distingue esta analogía del razonamiento por analogía tal como se entendía en la época de Darwin. 
dejar descendencia. De dos animales caninos, en tiempo de escasez y de hambre, puede decirse verdaderamente que luchan entre sí por conseguir alimento y vivir. Pero de una planta en el límite de un desierto se dice que lucha por la vida contra la sequedad, aunque fuera más propio decir que depende de la humedad. De una planta que produce anualmente un millar de semillas, de las que, por término medio, sólo una llega a la madurez, puede decirse con más exactitud que lucha con las plantas de la misma clase y de otras que ya cubren el suelo. El muérdago depende del manzano y de algunos otros árboles; mas sólo en un sentido muy amplio puede decirse que lucha con estos árboles, pues si creciesen demasiados parásitos en el mismo árbol, éste se extenúa y muere; pero de varias plantitas de muérdago que crecen muy juntas en la misma rama, puede decirse con más exactitud que luchan entre sí. Como el muérdago se disemina por los pájaros, su existencia depende de éstos, y puede decirse metafóricamente que lucha con otras plantas frutales, tentando a los pájaros a devorar y así diseminar sus semillas. En estos diversos sentidos, que se relacionan entre sí, empleo por razón de conveniencia el término general de «lucha por la existencia.» (OS, 50; OE, 102-103, Las cursivas son mías. Para mantener la uniformidad con el texto inicial mantengo «término» en lugar de «expresión», que figura en la traducción citada).

Este texto, que ya figuraba en la primera edición de OS (Darwin, 1859, 6263), puede leerse perfectamente en dos columnas, figurando en una de ellas los ejemplos de las expresiones metafóricas (falsas) y, en la otra en paralelo, las expresiones literales (verdaderas). La oposición fundamental que encabeza y orienta la lectura en dos columnas está en la oposición luchaldependencia.

La lucha por la existencia es la forma «falsa» en que se expresan las dependencias ecológicas «verdaderas» que caracterizan las relaciones de los organismos con sus condiciones de existencia. Limoges, (1970) y Tort (1996, Vol. 3, artículo «selection naturelle») han insistido en que este capítulo III es el capítulo ecológico de OS, señalándose, con razón, el carácter «ecológico» del principio darwiniano de SN (Limoges (1970), Tort (1996)). Y si, como el propio Darwin reconoce, las dependencias (relaciones e interacciones) de unos seres respecto de otros (bióticos o abióticos) son los referentes literales de «selección natural», ecológicamente entendida, cuya metáfora es «lucha por la existencia» (a la que llamaré de ahora en adelante $\mathrm{SN}_{1}$ ), entonces es posible conciliar un principio teórico general, procedente de la teoría maltusiana, con los hechos de coexistencia 
de especies diferentes en determinados territorios. En su artículo antes mencionado del diccionario por él dirigido (Cf. Tort, Vol. 3, 3933-35), ofrece Tort un esquema en diez puntos de la estructura argumentativa de OS. Los puntos 6 y 7 enfrentan una tesis general (6): la capacidad natural de los representantes de cualquier especie, animal o vegetal, de ocupar totalmente los territorios disponibles, y un hecho (7) (repetido, "casi general») que parece ir contra la tesis: la existencia casi universal de equilibrios naturales caracterizados por la coexistencia, en un mismo territorio, de representantes de múltiples especies. Darwin lo formula así:

No hay ninguna excepción a la regla de que todo ser orgánico aumenta naturalmente en progresión tan elevada que si no es destruido, pronto estaría la Tierra cubierta por la descendencia de una sola pareja.» (OS, 50-51; OE, 103. Las cursivas son mías).

Aunque se sustituya «la Tierra» por "un territorio dado», la observación muestra justamente que la morfología básica (MB), el modelo previsto en el principio, no se cumple, es decir, que las diferentes morfologías observadas (MO) se desvían de MB. Para explicar las MO, es necesario introducir las "causas» de la desviación (Cf. Álvarez, 1994). Esa conjunción de causas que permiten, en principio (de hecho, ya es otra cuestión), reconstruir las $\mathrm{MO}$, es $\mathrm{SN}$ en su versión interactiva denominada «lucha por la existencia», es decir, $\mathrm{SN}_{1}$.

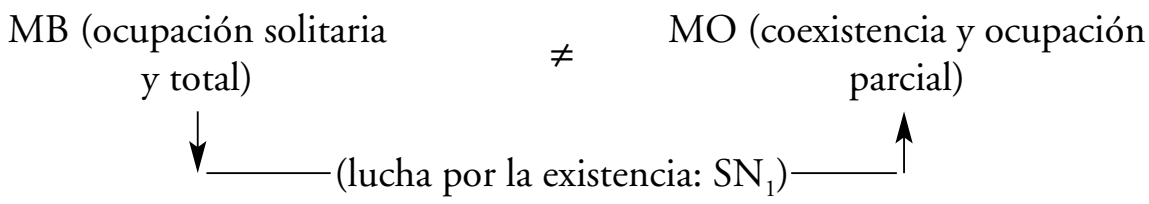

Darwin caracteriza esa conjunción de causas que permiten la «explicación» de las MO como «los obstáculos» que corrigen (check) el aumento de las poblaciones: «[l]as causas que contrarrestan la tendencia natural de cada especie a aumentar son de lo más oscuro [...] No sabemos exactamente cuáles sean los obstáculos, ni siquiera en un solo caso» (OS, 53; OE, 106).

La lucha por la existencia $\left(\mathrm{SN}_{1}\right)$, que hace en $\mathrm{SN}$ el paralelo de la acción humana en SA, es «verdaderamente» el conjunto de relaciones de dependencia entre los miembros de los diferentes organismos que coexisten en un territorio. En este marco del conjunto de restricciones u obstáculos al aumento de las pobla- 
ciones quedan subsumidas, inyectadas, las poblaciones humanas de Malthus de forma semejante a como quedaba subsumida SA en SN. El capítulo III de OS es el lugar en el cual la metáfora de la "lucha» se convierte en la representación de la(s) (inter)dependencia(s) en el marco de los sistemas ecológicos, cuando la analogía se transforma de impropia en propia, de metafórica en conceptual. Como consecuencia de esta transformación, se evita la personificación de la naturaleza, que Darwin entiende como «la acción conjunta y el producto de muchas leyes naturales». (OS, 63, OE, 116). Los partidarios de la naturalización de las ciencias sociales encontrarían aquí brasas que arrimar a sus sardinas, en contra de la tesis de una proyección antropomórfica del ensayo maltusiano sobre la naturaleza por parte de Darwin. La subsunción de la lucha particular de una especie en la sociedad, procedente de Malthus, en una lucha generalizada en el conjunto de las especies en la naturaleza, no trae consigo únicamente una diferencia de extensión, sino también una inversión de la perspectiva, muy útil para dar una lectura apropiada de otras metáforas darwinianas que se expresan en el empleo repetido de las expresiones "gobierno" (polity) o «economía» (economy) de la naturaleza refiriéndose a $\mathrm{SN}_{1}{ }^{19}$.

En el capítulo IV se ofrece la segunda metáfora asociada, esta vez como sustitutiva de «selección natural», como otra expresión "conveniente», tomada esta vez del filósofo Herbert Spencer. La expresión no apareció en la primera edición de OS, y fue usada inicialmente en (Darwin, 1868, Vol. 1, 6) y posteriormente adoptada en la quinta edición de OS (1869). En el capítulo III de OS aclara Darwin, de pasada, cuando ofrece la acepción $\mathrm{SN}_{1}$, que

[h]e llamado a este principio, por el cual toda variación ligera, si es útil, se conserva, con el término de Seleccción Natural, a fin de señalar su relación con la facultad de selección del hombre. Pero la expresión frecuentemente empleada por el Sr. Herbert Spencer de la Supervivencia de los más Aptos es más veraz (accurate) $\mathrm{y}$, a veces, igualmente conveniente. (OS, 49; OE, 101-02. Traducción modificada. Las cursivas son mías. He puesto «veraz» en vez de "exacta», porque se ajusta mejor a la oposición darwiniana entre verdadero y falso)

19 En la traducción citada suele vertirse tanto "polity» como «economy» por «economía». Es en este contexto del gobierno o economía de la naturaleza donde las especies, variedades, etc. encuentran su lugar (Cf. Sterrett, 2002). Ésta sería la base para buscar en Darwin un posible apoyo textual para el principio de la construcción de nichos, como complementario del de la selección natural. 
Como en Álvarez (2000), abreviaré esta metáfora asociada y el principio anteriormente formulado con « $\mathrm{SN}_{2}$ ". Al comenzar el capítulo IV, Darwin se pregunta: «¿Cómo actuará la lucha por la existencia [...] en lo que se refiere a la variación»? (OS, 62; OE, 115). Esto es, qué resultados produce y cómo opera el mecanismo causal sobre las variaciones, a las que tampoco cabe asignarle limitación alguna en principio, pues las relaciones ilimitadamente complejas de $\mathrm{SN}_{1}$ "[...] causan una complejidad infinita en la estructura, constitución y costumbres» (OS, 102; OE, 156) de los seres vivos. Así como, a falta de $S N_{1}$, la descendencia de una pareja ocuparía todo el espacio, en este caso, si nada lo impidiese, las variaciones crecerían sin límite. Éste sería también un supuesto contrafáctico que, sin embargo, Darwin no formula de manera explícita. Es un hecho, por una parte, que hay variaciones útiles, como se ha mostrado en la SA, y sería inconcebible que en un conjunto ilimitado de variaciones "no se hubieran presentado nunca variaciones útiles al propio bienestar de cada ser» (OS, 102; OE, 156. Traducción modificada).

Pero si alguna vez ocurren variaciones útiles a cualquier ser orgánico, los individuos así caracterizados tendrán seguramente las mejores probabilidades de conservarse en la lucha por la vida, y por el poderoso principio de la herencia, éstos tenderán a producir descendencia con caracteres semejantes. A este principio de conservación o supervivencia de los más aptos, lo he llamado selección natural (OS,102-103; OE, 156. Las cursivas son mías).

En tanto que diferencial, $\mathrm{SN}_{2}$ es (como exigía el modelo explicativo para $\mathrm{SN}_{1}$ ) también un principio de destrucción, de extinción. La acción selectiva lo es en dos sentidos: en el de la supervivencia de los más aptos y en el de la extinción de los menos aptos ${ }^{20}$.

La expresión «supervivencia de los más aptos» tiene, frente a la de «lucha por la existencia», el sentido de un resultado, a saber, que los mecanismos causales de $\mathrm{SN}_{1}$ producen los diferentes estados y procesos de coexistencia de las distintas formas

20 «Debido a la elevada progresión geométrica de aumento de todos los seres orgánicos, cada área está ya completamente provista de habitantes, y de esto se sigue que así como las formas favorecidas generalmente aumentan en número, las menos favorecidas generalmente disminuirán y llegaran a ser raras. La rareza, según nos enseña la geología, es precursora de la extinción. «(OS, 85; OE, 138, Las cursivas son mías). 
aludidos con «la supervivencia de los más aptos». Aunque Darwin distingue, incluso en los títulos de los capítulos III y IV, $\mathrm{SN}_{1}$ de $\mathrm{SN}_{2}$, están continuamente entrelazándose ambas acepciones de «selección natural». Ello no obsta para que sea posible marcar una mayor asociación entre $\mathrm{SN}_{1}$ y mecanismos causales interactivos, así como entre $\mathrm{SN}_{2}$ y los estados y procesos resultantes de la acción de $\mathrm{SN}_{1}$ sobre las variaciones dadas en cada momento. Es posible, simplificando al extremo, ilustrar la relación entre $\mathrm{SN}_{1}$ y $S N_{2}$ con relación a variaciones $\mathrm{v}_{1}, \mathrm{v}_{2}, \ldots \mathrm{v}_{\mathrm{n}}$, como sigue: (1) $\mathrm{SN}_{2}$ $=\mathrm{SN}_{1}\left(\mathrm{v}_{1}, \mathrm{v}_{2}, \ldots, \mathrm{v}_{\mathrm{n}}\right)$.Y, habida cuenta de que se trata de un proceso recurrente, ha de añadirse la condición (2) $\left[\mathrm{SN}_{1}\right]_{\mathrm{t}+1}=\left[\mathrm{SN}_{2}\right]_{\mathrm{t}}$, que da como resultado la forma iterativa (3) $\left[\mathrm{SN}_{1}\right]_{\mathrm{t}+1}=\left[\mathrm{SN}_{1}\right]_{\mathrm{t}}\left(\mathrm{v}_{1}, \mathrm{v}_{2}, \ldots \mathrm{v}_{\mathrm{n}}\right)$. En esta forma recurrente — salvando la distancia en tiempo y conocimientos-, se halla el atisbo de uno de los elementos incluidos en la posibilidad de la reforma del concepto de selección natural, que proponen Martínez y Moya (2009, 82 y ss.), incorporando la idea de causalidad descendente en relación con el concepto de $\mathrm{SN}$ como fuerza causal: «la causalidad es descendente cuando se toman en consideración lapsos sustanciales de tiempo (agrupando numerosas generaciones) como si fueran un solo instante» (Ibid., 83) .

Así se establece la unidad conceptual de la SN como la de una idea que abarca tanto los mecanismos causales $\left(\mathrm{SN}_{1}\right)$ por los cuales se alteran los estados de los sistemas en interacción, cambios de estado cuyas secuencias constituyen los procesos en los cuales tiende a producirse la conservación o la extinción, respectivamente, de los más o de los menos aptos $\left(\mathrm{SN}_{2}\right)$. Quedan también distinguidos la teoría causal y el principio de la SN. Todo ello en el doble lenguaje de las metáforas "falsas», pero convenientes, y la "verdad» de sus alternativas literales. Queda abierta la cuestión de cuál es, en cada caso, la expresión verdaderamente literal, si tal cosa existe.

\section{La selección natural como principio explicativo y como idea regulativa}

Continúo el análisis de la teoría (y el principio) de la $\mathrm{SN}$ desde un punto de vista metodológico. Puedo decir con más razón que Bowler $(2005,25)$, lo que él dice, cuestionablemente, de sí mismo: «nunca me he considerado un verdadero especialista en Darwin». Aun así voy abordar la idea de SN desde el punto vista de mi manera entender la explicación científica y sus modelos, en conexión con las relaciones entre su aplicación científica y su interpretación filosófica. 


\subsection{La SN en el circuito de la explicación científica}

Sobre el modo de razonar o el método empleado por Darwin se ha escrito, como sobre su contexto cultural, mucho, y más que vendrá. Ghiselin (1983) festejó el «triunfo del método» hipotético deductivo de Darwin, frente al supuesto inductivismo que el propio Darwin pretendía seguir tras las huellas de Whewell y Herschel, sobre todo en la búsqueda de las «verdaderas causas» del último (Cf. Gildenhuys, 2004). Diversos pensadores evolucionistas nos han proporcionado el esquema del «long argument» en términos de hechos e inferencias. Huxley (1965) ofrece un esquema de cinco pasos (tres «hechos» y dos «deducciones» a partir ellos). Tort (1996) resume la estructura argumentativa de Darwin en OS, conducente a la formulación de la teoría causal de SN, mediante un listado de diez pasos, entre los que coexisten observaciones, inducciones, deducciones, un ejemplo y la formulación de la hipótesis de la SN. Coincidiendo con la distinción de Sober (2009), Gayon (2009) distingue las dos caras del planteamiento darwiniano: la teoría del ancestro común y la de la $\mathrm{SN}^{21}$. En este apartado presentaré lo que he llamado el circuito de la explicación cientifica (Álvarez, en prensa), que me servirá para situar la hipótesis (a la postre principio) de la SN.

En Álvarez (1994) presenté un modelo de explicación científica, llamado reconstructivo-naturalista, en el cual las morfologías observadas (MO) se reconstruyen como "desviaciones» de las morfologías básicas (MB) — modelos, tipos ideales, etc.- - construidos sobre la base de determinadas leyes y supuestos adicionales - recurriendo a la intervención de leyes y/o factores de corrección que gobiernan esas desviaciones. $\mathrm{Al}$ tratar de $\mathrm{SN}_{1}$ ya se utilizó este modelo para representar la reconstrucción de la coexistencia de especies como una desviación de la tendencia de cada especie a ocupar, si no hay otras que lo impidan, todo el territorio (vid. supra).

El principio de inercia de Newton presenta una $\mathrm{MB}$ - el movimiento rectilíneo y uniforme- que contempla como excepción lo que es la regla, a saber, la existencia de otros cuerpos que, por la fuerza de atracción, desvían al primer cuerpo de su trayectoria, paso a paso, obteniéndose una trayectoria cerrada —una

${ }^{21}$ No tengo espacio para entrar en el detalle, ni para reproducir el excelente esquema que presenta Gayon (2009, 331). 
MO_ como desviación de la recta (Cf. Álvarez, 1994, 299). Aquí se distinguen dos tipos de leyes: las de tipo 1, que sirven para construir o expresar (como el principio de inercia) una $\mathrm{MB}$, y las de tipo 2, que sirven para reconstruir las $\mathrm{MO}$ como desviaciones de la MB (segunda ley de Newton, ley de la gravitación universal). Aunque ambos tipos de leyes, en cada explicación científica, requieran a veces supuestos auxiliares las primeras y factores de corrección las segundas, resta que la ley —o como, decía Kant (1890), lo universal— está dada, y su aplicación consiste en subsumir en ella los casos particulares. En versión neopositivista esto condujo al modelo de la ley subsuntora (covering law) de Hempel, que es un modelo inferencial ${ }^{22}$, cuya variante deductiva (N-D), a pesar de las diferencias teóricas y cronológicas, comparte con Kant la idea del procedimiento (Cf. Álvarez, 1998, 64). Pero ese mismo Kant, como es sabido, se preguntó qué ha de hacerse cuando sólo está dado lo particular. Su respuesta es que ha de buscarse, mediante la reflexión, el universal correspondiente, aunque ese universal sea solamente una forma que unifica los particulares dados en el marco de una ciencia empírica. Esa es la función que desempeña la idea de finalidad respecto de los conocimientos de los seres vivos en Kant (1790). En situación de carencia de leyes hay que formular hipótesis que puedan dar lugar a las $\mathrm{MB}$ con que comienza la explicación propiamente dicha. A continuación completo el modelo reconstructivo-naturalista insertándolo, como en Álvarez (en prensa), en el circuito de la explicación científica.

Cuando no hay leyes disponibles, la explicación no puede consistir en subsumir los casos en ellas y llevar a cabo a continuación la correspondiente reconstrucción, sino en buscar hipótesis alternativas, cada una de las cuales es un candidato rival entre otros y se procede posteriormente a una evaluación comparativa de las hipótesis $\left(\mathrm{H}_{\mathrm{i}}\right)$ rivales, que debe llevar a una inferencia hacia la mejor explicación $n^{23}$. Esta inferencia como tal no es una explicación. De ser posible, el circuito debe completarse con la utilización de la hipótesis para construir la correspondiente $\mathrm{MB}$ por medio de leyes de tipo 1, hecho lo cual habría que reconstruir la $\mathrm{MO}$ como una desviación MR de MB, por medio de leyes de tipo 2. En esquema, queda así resumido:

22 Para una crítica del modelo de N-D de Hempel, véase Álvarez (1994, 289-298).

23 El libro clásico sobre el tema es Lipton (2004). 


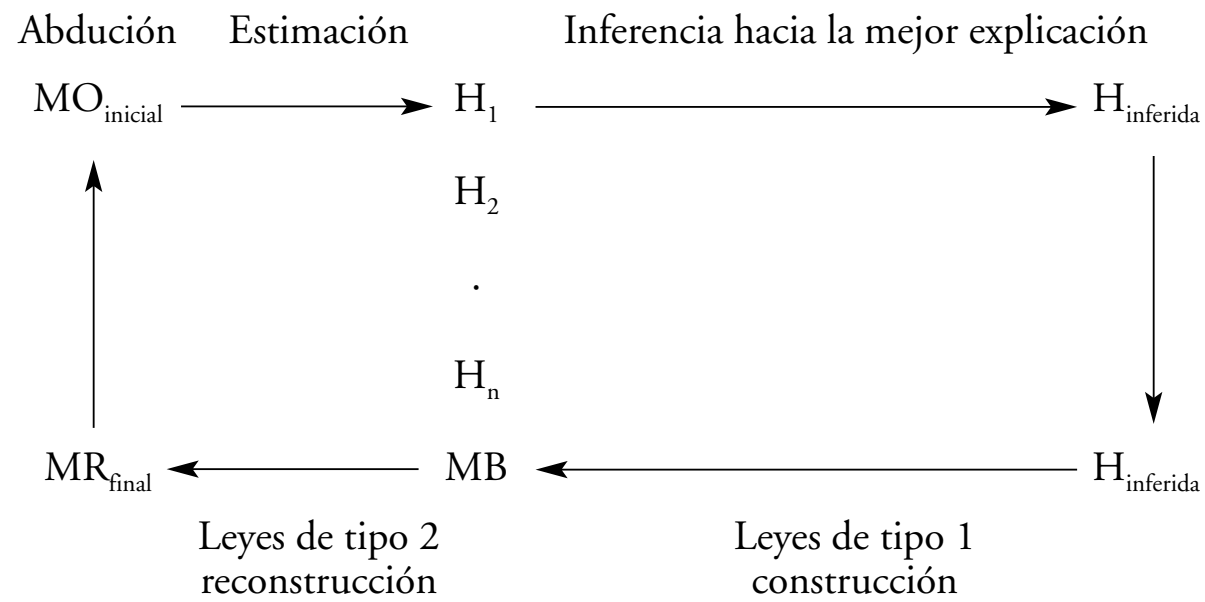

Peirce dio el nombre de «abducción $»^{24}$ a la forma de inferencia que extrae de o alcanza a partir de un conjunto de datos una hipótesis explicativa. Si acercamos esta noción a la del primer curso del circuito, la abducción realizada constaría de una estimación (la Beurteilung ${ }^{25}$ de Kant) de los datos como expresión de un orden y de una elección de la mejor de entre las hipótesis explicativas (en virtud de los criterios más solventes del conocimiento científico del momento) ${ }^{26}$. Si es factible, el progreso desde la $\mathrm{H}_{\text {inferida }}$, por medio de leyes de tipo 1, a una $\mathrm{MB}$, a partir de la cual, mediante leyes de tipo 2, se pudiera reconstruir una MR (final) » MO (inicial), serviría de confirmación —en el grado correspondientede la hipótesis elegida.

Como puede apreciarse, el modelo reconstructivo-naturalista es el curso inferior - de derecha a izquierda - del circuito explicativo, cuyo primer curso es abductivo, en vez de inductivo. El circuito que aquí se presenta completa el círculo meto-

${ }^{24}$ Peirce distinguió tres clases de inferencia: deducción, inducción y abducción. La forma abductiva fue llamada en primer lugar hipotética, después abductiva, después retroductiva y sólo en una etapa posterior abductiva de forma consistente (Cf. Fischer, 2001).

${ }^{25}$ Kant $(1790, \$ 61)$ utilizó este término para designar la estimación de los hechos de la naturaleza, en particular los fenómenos orgánicos, según la analogía teleológica que, a falta de leyes generales dadas, introduce en ellos un orden, al menos desde el punto de vista heurístico.

26 Aquí la abducción abarca tanto la estimación como la inferencia hacia la mejor explicación. Paavola (2006) distingue dos ideas de inferencia abductiva. Una asociada a la «lógica del descubrimiento», procedente de Hanson, y otra, procedente de Harman y a su caracterización de la inferencia hacia la mejor explicación. 
dológico que realmente no es recorrido una sola vez, sino que ha de suponerse en continua revisión en virtud de nuevos datos: la abducción es no monótona.

En su esquema metodológico del argumento darwiniano, Huxley (1965) apunta a que la evolución es "un hecho» que requiere explicación. Más que un hecho, Darwin acumula un corpus empírico de diversa procedencia que forma una de las caras del la teoría darwiniana. Como puntualiza Gayon $(2009,324)$ :

Desde finales del siglo XIX, se ha distinguido convencionalmente el «hecho general de la evolución» de su «explicación». Estas expresiones son, sin embargo, engañosas, porque lo que se llama «hecho general de la evolución» es ya, en realidad, una construcción teórica.

Darwin no tenía leyes - universales - dadas en que subsumir los hechos de toda índole en los diferentes registros de la morfología, la etología, la biogeografía, la paleontología, la ecología, la embriología, la taxonomía, etc. (ni siquiera algunos de estos nombres existían aún). Su entorno filosófico se caracterizaba por el inductivismo concurrente (consilient) de Whewell y el análisis subductivo ${ }^{27}$ en busca de las verae causae de Herschel. La historiografía posterior ha identificado, con Ghiselin (1983) a la cabeza, a Darwin con un practicante ejemplar del método hipotético deductivo. Más recientemente se multiplican los defensores de la abducción, en tanto que «lógica del descubrimiento», como procedimiento por el cual Darwin introdujo la hipótesis de la SN. (Cf. Kleiner, 2003; Paavola, 2004). Me atendré a poner en la forma del circuito explicativo anterior el planteamiento de Darwin.

$\mathrm{Al}$ inicio de la «Introducción» de OS declara Darwin:

Cuando iba como naturalista a bordo del Beagle [...] me sorprendieron mucho ciertos hechos en la distribución de los seres orgánicos que viven América del Sur, y las relaciones geológicas entre los habitantes actuales y los pasados de aquel continente. Estos hechos $[\ldots]$ parecian arrojar alguna luz sobre

27 «Los fenómenos complejos, en que varias causas operan a la vez, en concurrencia, oponiéndose o independientes unas de otras, produciendo un efecto compuesto, pueden simplificarse subduciendo el efecto de todas las causas conocidas [...] dejando, pues, por decirlo así, un fenómeno residual que ha de ser explicado» (Herschel, citado por Gildenhuys, 2004, 595). El mismo Gildenhuys declara a continuación que «Darwin trata la producción de variedades en estado doméstico como un fenómeno complicado que requiere el uso de la subducción en el primer capítulo del Origen» (Ibid). 
el origen de las especies [...] A mi regreso a casa se me ocurrió, en 1837, que acaso podría aclararse algo de esta cuestión acumulando y reflexionando pacientemente sobre toda clase de hechos que pudiesen tal vez tener alguna relación con ella. Después de cinco años de trabajo, me permití discurrir especulativamente sobre el asunto y redacté unas breves notas [...]»(OS, 1; OE, 55. Salvo el nombre del barco, las cursivas son mías).

El texto de Darwin nos da la entrada, porque «se dice que la inferencia abductiva parte de fenómenos anómalos o algo sorpendentes» ${ }^{28}$ (Paavola, 2004, 274). La paciente acumulación de hechos relacionados y la reflexión sobre ellos, le permitió discurrir especulativamente sobre el asunto. En el circuito de la explicación, a partir de hechos sorprendentes (MO) relacionados con el origen de las especies, a los que se agregan muchos más que se relacionan con el asunto y se reflexiona pacientemente - la estimación del circuito explicativo- sobre ellos, hasta discurrir especulativamente, «libremente» sobre el asunto. El resultado de esa especulación discursiva es elegir una hipótesis, entre otras (disponibles o «inventadas»: $\mathrm{H}_{1}, \mathrm{H}_{2}, \ldots \mathrm{H}_{\mathrm{n}}$ ) que pueda servir para conducir una explicación de la cuestión mejor que las de las hipótesis alternativas.

En las investigaciones científicas está permitido inventar cualquier hipótesis, y si ésta explica diversas clases grandes e independientes de hechos, se eleva al rango de una teoría bien fundada. [...] El principio de selección natural puede considerarse como una mera hipótesis, pero que se convierte en probable en cierto grado por lo que sabemos positivamente de la variabilidad de los seres orgánicos en estado de naturaleza [...] Ahora bien, esta hipótesis puede ser contrastada [...] intentando ver si explica varias clases grandes e independientes de hechos [...] Si el principio de selección natural explica realmente [...] grandes cuerpos de hechos, ha de ser aceptado. (Darwin, 1868, 8-9. Las cursivas son mías).

Darwin, ciertamente, fue paciente hasta el extremo, mientras crecía en él, como un abrumador huevo de kiwi (Cf. Quammen, 2007), la teoría de la SN. Si mi percepción es acertada, los años de acumulación de hechos y reflexiones sirvieron para construir su MO, a la que denominó «descendencia con modifi-

28 A Christaller le «sorprendía» que pareciera existir una relación no accidental entre el tamaño, el número y la distribución de los asentamientos urbanos que abastecen un territorio circundante. Este fue el punto de partida de su teoría de los lugares centrales. (Cf. Álvarez, 1991). 
cación», que se basa en un conjunto de conjeturas que, según Gayon (2009, 325) se agrupan en los capítulos IV (selección natural) y XIII (XIV de la $6^{a}$ edición) dedicado a la clasificación. Darwin ofreció un diagrama arborescente que sintetiza esas conjeturas en el capítulo IV: la $M O_{\text {inicial }}$ tiene su representación gráfica ${ }^{29}$. Tras «discurrir especulativamente», «inventó» la hipótesis de la SN como alternativa a la de la creación especial (CE) - la selección sobrenatural- de la teología «natural». Como señala Castrodeza $(2009,61)$ [1]a intención principalísima de Darwin [...] era demostrar que el fenómeno de la adaptación no estaba propiciado por la divinidad, sino por causas naturales». En Darwin $(1868,9)$, elige $\mathrm{SN}$ contra CE con esta justificación:

En la concepción corriente en que cada especie ha sido independientemente creada, no obtenemos explicación alguna de ninguno de estos hechos [la MO]. Sólo podemos decir que le ha placido así al Creador mandar que los habitantes pasados y presentes del mundo aparezcan en un cierto orden y en ciertas zonas; que ha imprimido en ellas las semejanzas más extraordinarias y que las ha ordenado en grupos subordinados a grupos. Pero con semejantes afirmaciones no adquirimos ningún conocimiento nuevo; no reunimos hechos y leyes; no explicamos nada. (Las cursivas son mías).

Elegida la hipótesis, se pone en marcha el desarrollo de la teoría causal de la $\mathrm{SN}$, analizado en la sección anterior y que culmina en el principio de la SN del capítulo IV. Con todo lo interesante que pueda ser la distinción de Sober (2009) entre la parte causal y la parte evidencial de OS, y también la de las dos caras o enfoques (les deux volets) de OS — descendencia con modificación y selección natural- de Gayon (2009), el texto darwiniano nos orienta. En el capítulo IV, el apartado en que figura el diagrama en árbol, se titula «Efectos probables de la selección natural, mediante la divergencia de caracteres y la extinción, sobre los descendientes de un ancestro común» (OS, 90; OE, 143). Aquí se ve que las dos caras de OS no se suman, sino que se combinan ${ }^{30}$.

${ }^{29}$ Aunque parezca extraño, a pesar de los argumentos de Gayon (2009), que el árbol del capítulo IV sea considerado como la representación de la $\mathrm{MO}_{\text {inicial }}$, más adelante quedará clara la diferencia entre este árbol no representado y el diagrama del capítulo IV.

30 «[...] en lugar de describir la teoría de Darwin como evolución por selección natural, queda mejor descrita como ancestro común más selección natural» (Sober, 2009, 53. Cursivas del original). Está mucho más acertado en Sober (2008, xvii. Cursivas del original): «Los biólogos utili- 
El árbol de Darwin se lee verticalmente hacia arriba según el tiempo geológico y horizontalmente en términos del espacio "ecológico», del espacio configurado según la economía (economy) o gobierno (polity) de la naturaleza, en la que unas variedades y especies resultantes ocupan su lugar y otras se extinguen. La MB tiene su diagrama, el diagrama general de un árbol como el descrito. También la $\mathrm{MO}_{\text {inicial }}$, en cuanto ligada a la idea de la descendencia con modificación, solidaria de ancestros comunes — varios o uno-, también la tiene implícita o, mejor dicho, tiene muchas implícitas dependiendo de a qué grupos de organismos esté referida. El diagrama genérico del capítulo IV es el «árbol de los árboles» ${ }^{31}$ y como tal $\mathrm{MB}$ no ha de compararse con la $\mathrm{MO}_{\text {inicial }}$, sino que ha de servir de base para una morfología reconstruida (MR), lo más próxima posible de $\mathrm{MO}_{\text {inicial }}$. Pero esta reconstrucción ya no puede hacerse recurriendo a la SN como teoría que explica las adaptaciones. Hay que introducir leyes de tipo 2 o factores de corrección que hoy, cuando se sabe mucho más que Darwin, son los acompañantes necesarios de la SN en la ruta de la evolución. Darwin ya puso los suyos, el uso y el desuso, las correlaciones de crecimiento, etc., que son bien conocidos. Son los otros principios «no principales» de la evolución, que corrigen los resultados de la $\mathrm{MB}$ en que culmina la teoría causal de la SN. Los pluralistas de los principios sostienen, frente a lo que consideran fundamentalismo seleccionista, que el principio de SN es «un principio supremo (verdaderamente un primus inter pares), pero argumentan a continuación que un conjunto adicional de leyes, tanto como un extenso papel de las impredecibles contingencias históricas, han de ser invocados también para explicar las formas (patterns) y regularidades básicas de las trayectorias de la vida» (Gould $(1997,1)$. Sin embargo, la noción de primus inter pares es confusa, pues pone "dentro de» $\mathrm{y}$ «entre» los miembros de un conjunto lo que está en un conjunto diferente de otro - el de los falsos pares. El principio de $\mathrm{SN}$ es una ley de tipo 1 que sirve para construir la $\mathrm{MB}^{32}$, mien-

zan información acerca de la ascendencia común para poner a prueba hipótesis acerca de la selección natural. Y las inferencias acerca de la ascendencia se apoyan a menudo en información acerca de cómo han evolucionado varios rasgos. Las dos partes de la representación (picture) darwiniana son lógicamente independientes, pero son metodológicamente interdependientes». Precisamente, el planteamiento que hago es metodológico.

31 Comentando este diagrama del capítulo IV dice Darwin: «[...] no supongo que el proceso continúe siempre tan regularmente como está representado en el diagrama [...] (OS, 91; OE 145). Esto distingue las $\mathrm{MO}$ —árboles empíricos — de la $\mathrm{MB}$ —árbol genérico del diagrama-.

32 No considero ya a SN, como en Álvarez (2000), un «metaprincipio», tal como figura en la conclusión de aquel trabajo. 
tras que los otros principios y las llamadas contingencias son, respectivamente, leyes de tipo 2 o factores de corrección que sirven para reconstruir una $\mathrm{MR}_{\text {final }}$, que sí debe ser comparable con la $\mathrm{MO}_{\text {inicial. }}$. Si los resultados de la comparación no son aceptables, hay que volver atrás e ir revisando, en primer lugar, las bases de la reconstrucción y, todo es posible, hasta incluso la propia hipótesis «inventada». No parece que ese sea el caso con la teoría darwiniana, aunque pase a veces por el quirófano para nuevas síntesis.

Desde el punto de vista de la explicación científica, la teoría darwiniana de la evolución se ajusta razonablemente bien al circuito de la explicación científica o, para invertir el planteamiento, el circuito de la explicación recoge bien el método darwiniano en un recorrido que une descubrimiento y justificación.

\subsection{La SN entre las ideas filosóficas}

La teoría de la selección natural, discutida en su nacimiento, eclipsada aproximadamente desde 1870 hasta cerca de 1930, renacida con la «síntesis moderna», alimentada por la genética molecular y la evo-devo, y a la espera de la nueva síntesis del siglo XXI, ha tenido relaciones importantes con la filosofía. Nació en el ambiente de la filosofía inductivista del siglo XIX, pero, como se ha visto, tras pasar por ejecución del método hipotético deductivo ${ }^{33}$ (pensado en el contexto de justificación), admite bien tanto el tratamiento abductivo con relación a la formulación de hipótesis como las explicaciones por reconstrucción de las MO como desviaciones de una MB. En esto se ajusta a un modelo explicativo bien conocido y utilizado habitualmente.

Pero por qué tantos filósofos y teólogos — profesionales y aficionados — siguen apoyando o combatiendo una "filosofía oculta o implícita» en el pensamiento darwiniano. Según Ghiselin, (1983) Darwin eliminó el esencialismo ontológico de las especies y la teleología del mundo biológico: no sólo la teológica de Paley, sino también la metodológica de Kant. Quiero considerar aquí estas dos sustituciones.

33 Ruse $(2008,75)$, se pregunta qué partes de OS se ajustan al esquema hipotético deductivo, y responde: «Desde luego, si exigimos una definición estricta, no muchas». 
La primera, la teoría causal de la SN establece su nivel de resolución en el par conjugado variedades/especies. He definido (entre otros lugares, en Álvarez 1988a, 48 y ss.) el nivel de resolución de una teoría científica como el par conjugado de dos escalas o niveles contiguos de su dominio de objetos, entre los cuales discurren las explicaciones. El concepto de nivel de resolución es un concepto filosófico ontológicamente fundado, pero no dado de antemano, sino metodológicamente establecido, que se alcanza en el desarrollo histórico de la ciencia. El par conjugado variedades/especies juega ese papel en la teoría darwiniana. Para ello, sin embargo, fue necesario establecer una articulación entre los niveles del dominio objetivo de la teoría. En el resumen del capítulo II de OS, Darwin establece claramente la relación y el tránsito entre variedades y especies, que permite unificar su origen con relación al mecanismo de la SN.

En conclusión, las variedades no pueden distinguirse de las especies, excepto: primero, por el descubrimiento de formas de enlace intermedias, y, segundo, por cierta cantidad indefinida de diferencia entre ellas [...] la cuantía de diferencia que se considera para dar a cualquiera de las dos formas [consideradas] la categoría de especie, no se puede determinar [...] las especies de los géneros grandes presentan una analogía muy intensa con las variedades. Y podemos comprender claramente estas analogías, si las especies existieron en otro tiempo como variedades y se originaron de este modo; mientras que estas analogias resultan completamente inexplicables si las especies son creaciones independientes (OS, 47; OE, 100. Las cursivas son mías).

La analogía es perfectamente clara:

$$
\text { variedades : especies :: SN(variedades) : SN(especies) }
$$

y se basa en un principio de continuidad entre variedades y especies: «[...] las especies no son más que variedades muy acusadas y permanentes, y [...] cada especie existió primero como variedad[...]»(OS,413; OE, 463). Esta es la base del gradualismo filético. De ahí se extrapola hacia las categorías superiores. Los textos de Darwin contienen explícitamente la analogía variedad : especie :: especie : género. Esa extrapolación, asentada sobre la descendencia — una conexión material que prolonga más allá el principio de continuidad utilizado sólo localmente en el establecimiento del nivel de resolución variedades/especies — confiere a la evo- 
lución su unidad global en un encabalgamiento de categorias que unifica el mundo de la vida ${ }^{34}$.

La SN sustituye tanto la CE de la teología natural como la teleología que tenía, en la filosofía de Kant, el carácter de una idea regulativa o máxima para la investigación del mundo de la vida, sin atribuir ontológicamente la finalidad a los procesos biológicos. Kant $(1790, \$ 80)$ ingenió una fórmula en la cual se subordina la máxima mecanicista a la máxima teleológica, pero imponiendo la necesaria adjunción de la primera a la segunda. Los organismos son, según este planteamiento, fines de la naturaleza, que no pueden explicarse como meros productos de la misma, aunque lo sean. Por ello, la subordinación y adjunción mencionadas caracterizan una metodología de la investigación en que se subordinan las causas eficientes a las causas finales, sin que aquéllas sean eliminadas. Una especie de metodología con la que Kant hace de necesidad virtud, aún alentando una débil esperanza de una ciencia biológica ${ }^{35}$. Darwin invirtió la relación kantiana subordinando la máxima teleológica a la mecanicista, adjuntando forzosa e implícitamente la primera a la segunda. Con ello, inversamente a Kant, defiende la explicación de los fines de la naturaleza como productos de la naturaleza, a saber, las adaptaciones como productos de la SN. Kant concebia la adaptación —-metodológicamente - desde la finalidad, mientras que Darwin convierte la finalidad -ontológicamente-en adaptación. Hasta aquí no me he referido directamente a la adaptación, algo que puede parecer extraño a quien haya llegado hasta este punto. Pero en mi no tan largo argumento, era necesaria la exposición de la teoría causal de la SN, la identificación, como diferente de y como culminación de ella, del principio de SN y la presentación del largo argumento de Darwin en la forma del circuito de la explicación científica. Como profesor en asignaturas y cursos de Historia de la ciencia, he utilizado la estrategia de situar una ciencia en el marco de sus relaciones con la técnica y la filosofía a través de los textos clásicos. Por poner dos ejemplos textuales de las relaciones entre ciencia y filosofía: para la geometría griega, codificada en los Elementos de Euclides, está la concepción de la diánoia en los ejemplos de la línea dividida de La Repú-

${ }^{34}$ Que abandone el esencialismo no significa que el nominalismo pragmático (débil) sea incompatible con un realismo semántico asentado sobre la descendencia, aunque su representación conjuntista no sea nítida, sino borrosa. Para esta compatibilidad, véase Lewens (2007, 70 y ss.).

${ }^{35}$ He tratado en detalle este tema en Álvarez (1998). Aquí sólo cabe ver la inversión darwiniana de la relación entre finalidad y causalidad, propuesta por Kant. 
blica de Platón (Cf. Álvarez, 2005) como filosofía que reflexionaba sobre aquella geometría; para los Principios de Newton se dispone de los Principios metafisicos de la ciencia de la naturaleza de Kant (Cf. Álvarez, 1988b). Mi tercer gran texto era el OS de Darwin: no encontré nada parecido a pesar de la «industria darwiniana» y de todo el tiempo transcurrido. Puede que la progresiva especialización, que también afecta a la filosofía, o mi falta de erudición sea la causa, o las dos juntas. O puede, y esto es más arriesgado, que tuviera delante, como en La carta robada de Poe, lo que estaba buscando y no lo viera, porque la carta no se aparecía como tal, sino como un simple sobre. La carta estaba dentro. El filósofo buscado estaba envuelto por el sobre del científico llamado Charles Robert Darwin, «cazador, creacionista y naturalista» (Castrodeza, 2009, 34) en su juventud, y enfermo crónico, evolucionista y naturalista por partida doble en su madurez y ancianidad.

En el «Prólogo» a la séptima reimpresión del Treatise on Natural Philosophy de Lord Kelvin (Thomson \& Tait, 1923, 2) se lee:

El término Filosofía Natural fue utilizado por Newton, y todavía se usa en las universidades británicas para denotar la investigación de las leyes del mundo material y la deducción de resultados no observados directamente. La observación, la clasificación y la descripción de los fenómenos preceden necesariamente a la Filosofía Natural en cada especialidad de la ciencia natural. La primera etapa, en algunas ramas, se llama Historia Natural, y podría llamarse así, con igual corrección, en todas las demás. (Las mayúsculas de los nombres son del original; las cursivas son mías).

Digamos que Darwin, fuera o no «el Newton de la brizna de hierba», pasó, en su rama, a la segunda etapa con su teoría de la evolución por selección natural. Naturalista historiador terminó siendo naturalista filósofo, aunque "filósofo» abarque aquí tanto a científicos como a filósofos en la actual acepción de los términos, y la «filosofía natural de los organismos» contenía, a su manera, por analogía con los de Kant (Cf. Álvarez, 1988b), los principios «metafisicos» ${ }^{36}$ de la

36 En este punto es donde se embarullan todas las disputas entre científicos, filósofos y ontoteólogos. Los científicos darwinistas stricto sensu no querrían ni oír semejante palabra —la ciencia (ni ellos) no tiene nada que ver con la metafísica-, pura especulación filosófica ya superada por la ciencia que ellos practican: la SN es el principio científico que unifica todos los conocimientos 
Historia natural, que habrían de reemplazar los principios teológicos hasta entonces dominantes. Proporcionó, con la teoría de la SN, una explicación causal verosímil de la evolución de la vida en la Tierra y marcó con SN, como idea regula$t i v a^{37}$, las grandes líneas de un inmenso programa cuya ejecución no estaba en condiciones de prever en todos sus detalles científicos, filosóficos y tecnológicos. Precisamente por ello, el darwinismo posterior es algo más que la teoría darwiniana de la evolución por $\mathrm{SN}$, en la medida en que transforma la idea regulativa en condición constitutiva y norma directiva de nuestro conocimiento, pensamiento y acción, en la línea de lo que Castrodeza (2009) llama «darwinización del mundo». Pero eso sí, por la otra cara (también aquí hay dos), imponiendo a los seres humanos, productos de la naturaleza, «cuyos conceptos son los conceptos de la naturaleza sobre sí misma [...] la responsabilidad de la naturaleza en general y de la humana en particular» (Markl, 2003, 130). Por tanto, nos remite no sólo a la metafísica, sino también a la bioética, más allá del ámbito metodológico en que aquí se analiza la teoría darwiniana de la SN.

\section{A modo de conclusión}

Desde Darwin, la SN no ha dejado de ser un tema controvertido. En su propio creador osciló lingüísticamente entre lo metafórico y lo literal, metodológicamente pasó de hipótesis a principio y filosóficamente de idea regulativa a concepto metafísico. Para una noción, el recorrido por esa diversidad de registros no debe contemplarse como una multivocidad dañina, sino como la fecundidad de una idea que para algunos darwinistas se identifica con el darwinismo simpliciter o, más aún, junto a los principios de variación y herencia, como uno de los tres puntales de un darwinismo universal o, al menos, generalizado (Cf. Álvarez, por aparecer). Del nacimiento de Darwin a la actualidad han transcurrido doscientos años y la historia intelectual que comienza, básica y públicamente, hace ciento cincuenta con El origen de las especies está escrita en muchas claves. Pero

biológicos (Cf. Moreno, 2008). Los ontoteólogos (entre ellos los creacionistas y los defensores del diseño inteligente) rechazan precisamente cualquier metafísica naturalista por materialista (sea cual sea la manera en que entiendan esa palabra) y porque deja la vida humana sin sentido trascendente.

37 Que sirva la función regulativa no impide que, por su condición de principio explicativo, no tenga también que ver con la ontología (si se prefiere esta palabra a «metafísica», dada la historia de la metafísica dominante en la Universidad española de la posguerra, que tiene asociaciones poco asumibles para quienes se interesen por la ciencia). 
ninguna de las interpretaciones de ellas - y menos aun las más opuestas y combativas - puede considerar ni a Darwin, ni al evolucionismo que se declara su heredero, como un episodio al que le ha llegado su hora. Estamos muy lejos de que nadie pueda dedicarle un sic transit gloria mundi.

\section{Bibliografía}

ÁlvareZ, J. R., 1988a, Ensayos metodológicos, León: Universidad de León.

- 1988b, «The Underlying Structure of Kant's Metaphysische Anfangsgründe der Naturwissenschafti, en FunKe, G. \& SEEBOHM, T., Proceedings of the Sixth International Kant Congress (1985), Washington D.C.: The University of America Press, 1988, Vol. II/2, 49-64.

- 1991, La racionalidad hexagonal. La identidad cientifica de la teoría de los lugares centrales, León: Universidad de León.

- 1994, "Dos modelos de explicación científica: inferencias y reconstrucciones», Contextos, XII/23-24, 1994, 287-311, accesible en http://www3.unileon.es/dp/alf/ dosmod.pdf (Consultado 01/08/2009).

- 1998, «Una débil esperanza: la idea kantiana de una ciencia biológica», en ARANA, J. (ed.), Los filósofos y la Biologia, Themata 20, 1998, 49-66.

- 2000, "Analogías darwinianas: modelos y/o metáforas», en MorA, M.S. et al. Actas del III Congreso de la Sociedad de Lógica, Metodología y Filosofía de la Ciencia, San Sebastián: Universidad del País Vasco, 2000, 331-341.

- 2005, «La filosofía y las terceras culturas», Contextos, XXIII-XXIV/45-48 (20052006), 7-25, borrador accesible en http://www3.unileon.es/dp/alf/3acult.pdf. (Consultado 22/07/2009).

- en prensa, «Freedom of actions and their necessary outcomes: Fractal networks in geographic space», GonZÁLEZ, W. J, (ed.), Freedom and Determinism: Social Sciences and Natural Sciences.

- por aparecer, «De aquel Darwin tan singular al darwinismo universal: la problemática naturalización de las ciencias de la cultura», Ludus Vitalis, XVII, 32, 2009.

BOwLER, P.J., 2005, «Revisiting the Eclipse of Darwinism», Journal of the History of Biology, 38, 2005, 19-32. 
Castrodeza, C., 2009, La darwinización del mundo, Barcelona: Herder.

DARWIN, C., 1859, On the Origin of Species by means of Natural Selection, or The Preservation of Favoured Races in the Struggle for Life. London: John Murray.

- 1868, The Variation of Animals and Plants under Domestication, London: John Murray, 2 Vols.

- 1876, The Origin of Species by means of Natural Selection, or The Preservation of Favoured Races in the Struggle for Life, $6^{\text {th. }}$ ed., with additions and corrections, London: John Murray.

- 1985, El origen de las especies. Prólogo de F. Cordón, traducción de A. Froufe, Madrid: EDAF.

DAWKINS, R., 1983, «Universal Darwinism», en BENDALL, B.S (ed.) Evolution from

Molecules to Man, Cambridge: Cambridge University Press, 1983, 403-425.

DennetT, D. C., 1995, Darwin's Dangerous Idea. Evolution and the Meanings of Life, New York: Simon \& Schuster.

Eldredge, N., 2009, Darwin. El descubrimiento del árbol de la vida. Traducción de J. Barba y S. Jawerbaum, Madrid : Katz.

FISCHER, H. R., 2001, «Abductive Reasoning as a Way of Worldmaking», Foundations of Science, Vol. 6(4), 2001, 361-383,

GAYON, J., 2009, «Mort ou persistance du darwinisme? Regard d'un épistémologue», C.R. Palevol 8 (2009), 321-340.

Ghiselin, M.T.,1983, El triunfo de Darwin. Traducción de E. Pérez Sedeño, Madrid : Cátedra.

GiLdenhuys, P., 2004, «Darwin, Herschel and the role of analogy in Darwin's origin», Studies in History and Philosophy of Biological and Biomedical Sciences, 35, 2004, 593-611.

GinNOBILI, S., 2009, «Selección artificial, selección sexual, selección natural», accesible en http://santi75.files.wordpress.com/2009/06/seleccion-artificial-seleccion-sexualseleccion-natural.pdf (Consultado 08/07/2009).

Gould, S. J., 1997, «Evolution: The Pleasures of Pluralism», New York Review of Books, June 26, 1997, en http://www.stephenjaygould.org/reviews/gould_pluralism.html (Consultado 07/07/2009).

— 2002, The Structure of Evolutionary Theory, Cambridge (MA): Harvard University Press. 
HuXley, J., 1965, La evolución. Sintesis moderna. Traducción de F. Jiménez de Asúa, Buenos Aires: Losada.

Johnson, P. E., 1995, Proceso a Darwin. El porqué la teoría darwinista no es nada más que una teoría. Traducción de S. Escuain, Grand Rapids (MI): Editorial Portavoz.

KaNT, I., 1913 [1790], Kritik der Urtheilskraft, en Kants gesammelte Schriften, Vol. V, Reimer: Berlín, 165-485.

KlEINER, S. A., 2003, «Explanatory coherence and empirical adequacy: The problem of abduction, and the justification of evolutionary models», Biology and Philosophy, 18, 2003, 513-527.

Laland, K. L, Odling-Smee, J., Feldman, M.W. \& Kendall, J., 2009, "Conceptual barriers to progress within Evolutionary Biology», Foundations of Science, 14, (3), 2009, 195-216.

LEIBNIZ, G.W. 1978 [1678], «Quid sit idea», Die philosophischen Schriften, Hrsg. von C.I. Gerhardt, Hildesheim: G. Olms, Vol. VII, 263-264.

LEWENS, T. 2007, Darwin, London : Routledge.

Limoges, C., 1970, La sélection naturelle. Étude sur la première constitution d'un concept (1837-1859), París: Presses Universitaires de France.

LiPTON, P., 2004, Inference to the Best Explanation, $2^{\text {nd }}$ edition, London: Routledge.

MARKL, H., 2003, «Humanity and humanism. The bioethical perspective of human evolution», Current Science, 84 (10), 2003, 1309-1316, en http://www.ias.ac.in/currsci/ may252003/1309.pdf. (Consultado 20/07/2009).

Martínez, M. y MoYa, A., 2009, «Selección natural, creatividad y causalidad», Teorema, Vol. XXVIII/2, 2009, 71-94.

OrGEL, L., 1979, Los orígenes de la vida. Moléculas y selección natural. Traducción de E. López Thome, Madrid: Alianza Editorial.

PAAVOLA, S., 2004, "Abduction as a logic and methodology of discovery: the importance of strategies», Foundations of Science, 9(3), 2004, 267-283, accessible en http://www.helsinki.fi/science/commens/papers/abductionstrategies.html (Consultado 12/07/2009).

- 2006, "Hansonian and Harmanian Abduction as Models of Discovery», International Studies in the Philosophy of Science, 20(1), 2006, 93-108. 
Pievani, T. 2009, Creación sin Dios. Traducción de S. Schettin, Madrid: Akal.

Quammen, D., 2007, The Kiwi's Egg. Charles Darwin \& Natural Selection, London: Weidenfeld \& Nicholson.

Ruse, M., 2008, Charles Darwin. Traducción de E. Marengo, Madrid: Katz.

Russell, R.J., Stoegen, W. R. \& Ayala, F.J. (eds.), 1998, Evolutionary and Molecular Biology. Scientific Perspectives on Divine Action, Vatican City/Berkeley: Vatican Observatory/Center for Theology and Natural Sciences.

SANMARTín, J., 1987, Los nuevos redentores. Reflexiones sobre la ingeniería genética, la sociobiología y el mundo feliz que nos prometen, Barcelona: Anthropos.

SARKAR, S., 2007, Doubting Darwin? Creationist Designs on Evolution, London: Blackwell.

Sober, E., 1993 [1984], The Nature of Selection. Evolutionary Theory in Philosophical Focus, Chicago: The University of Chicago Press.

- 2008, Evidence and Evolution. The Logic behind the Science, Cambridge: Cambridge University Press.

— 2009, «¿Escribió Darwin el Origen al revés?», Teorema, Vol. XXVIII/2, 2009, 45-69.

STERrETT, S. G., 2002, «Darwin's analogy between artificial and natural selection: how does it go?», Studies in History and Philosophy of Biological and Biomedical Sciences, 33, (2002), 151-168.

Thomson, W. \& TAIT, P.G., 1923, Treatise on Natural Philosophy, $7^{\text {th. }}$ reprint, Cambridge: Cambridge University Press.

TORT, P. (Dir.), 1996, Dictionnaire du darwinisme et de l'évolution, París, Presses, Universitaires de France, 3 vols.

Young, R. M, 1985, Darwin's Metaphor: Nature's Place in Victorian Culture, Cambridge: Cambridge University Press.

- 1993, «Darwin's Metaphor and the Philosophy of Science» Science Culture, 16, 3: 375-403, 1993, accesible en http:/www.human-nature.com/rmyoung/papers/ paper8.html. (Consultado 07/07/2009).

Recibido: 17/08/2009

Revisado: 10/09/2009 